\title{
ENSINO, PESQUISA E EXTENSÃO UNIVERSITÁRIA SOB A ÓTICA DO DESIGN PARA INOVAÇÃO SOCIAL
}

\author{
TEACHING, RESEARCH AND UNIVERSITY EXTENSION FROM A PERSPECTIVE OFDESIGN \\ FOR SOCIAL INNOVATION
}

BEANY MONTEIRO, Dra. | UFRJ

\begin{abstract}
RESUMO
O artigo apresenta o relatório de uma pesquisa realizada durante o período de pós-doutorado cujo tema central de investigação são as práticas pedagógicas do Design para a Inovação Social, tendo como objetivo a estruturação da disciplina Design Ecossocial. Para desenvolver essa investigação nesse período partiu-se de uma reflexão sobre a criação e implementação do Programa de Pós-Graduação em Design da Escola de Belas Artes da Universidade Federal do Rio de Janeiro. O método de pesquisa baseou-se na associação das características intrínsecas e extrínsecas dos Objetos Intermediários de Concepção desse Programa com os instrumentos pedagógicos desenvolvidos durante a sua estruturação, e sobre o potencial desses instrumentos para participar associativamente na criação de novos Programas de Pós-Graduação no Brasil. Conclui-se que a preservação da autonomia dos conhecimentos gerados em contextos diversos, segundo o princípio de individuação dos Objetos Intermediários de Concepção, é potencialmente capaz de reduzir as desigualdades territoriais pelo fortalecimento de suas associações, e realizando assim um papel de coordenador das formas associativas.
\end{abstract}

PALAVRAS CHAVE: Design; inovação social; ensino; pesquisa; extensão

\begin{abstract}
This article presents the report of a research carried out during the postdoctoral program, which focused on the pedagogical practices used for teaching Design for Social Innovation, with the objective of structuring the course of Ecosocial Design, a class to be taught to undergraduate college students majoring in Design in Brazil. To develop the search in this period, it was initiated a reflection on the creation and application of the Postgraduate Program in Design at the School of Fine Arts of the Federal University of Rio de Janeiro. The research method was based on the combination of the intrinsic and extrinsic aspects of the intermediate objects that are need to the creation of this Program with the pedagogical instruments developed during its organization, and the potential of these instruments to participate associatively in the creation of new Graduate Programs in Brazil. It was determined that the preservation of the autonomy of the knowledge accrued in different contexts, according to the principle of individuation of the Intermediate Objects of Conception, is potentially capable of reducing territorial inequalities by strengthening their associations, and thus performing a coordinating role of associative forms.
\end{abstract}

KEY WORDS: design; social innovation; teaching; research; extension 


\section{INTRODUÇÃO}

Esse artigo apresenta uma reflexão realizada durante o período de pós-doutorado no ÉCO-LAB/ÉSAD-Écologie- Design - Numérique / École supérieure d'art et de design, Orléans, e Le Studium Loire Valley, Institute for Advanced Stud-ies, na França. Seu conteúdo trata especificamente das bases teóricas da pesquisa e de uma de suas possíveis aplicações, de acordo com o seu referencial teórico e metodológico.

Parte-se da experiência como membro do corpo científico que criou o Programa de Pós-Graduação em Design da Universidade Federal do Rio de Janeiro (PPGD/ EBA/UFRJ), e do aprofundamento da revisão bibliográfica, baseados na participação em diferentes conferências e seminários durante esse período (Monteiro in PIRES e GRIMALDI, 2020).

Uma reflexão sobre a criação e implementação do PPGD/EBA/UFRJ, e relacionada à pesquisa em curso: Ensino, pesquisa e extensão universitária sob a ótica do Design para inovação social, deu-se no sentido de identificar as características intrínsecas e as características extrínsecas dos Objetos Intermediários de Concepção desse Programa, com o objetivo de contribuir para uma possível participação do PPGD/EBA/UFRJ nas formas associativas, de acordo com a Portaria no 214, de 27 de outubro de 2017, da CAPES (COORDENAÇÃO DE APERFEIÇOAMENTO DE PESSOAL DE NÍVEL SUPERIOR) no Brasil.

Para tal, tomou-se como base a Disciplina Design Ecossocial, ministrada inicialmente com o nome de Objetos Intermediários de Concepção, desde 2017 (MER, JEANTET, TICHKIEWITCH, 1995). Foi proposto como abordagem as "formas associativas" de acordo com a Portaria no 214 da CAPES, cujos objetivos estão definidos no Artigo $2^{\circ}$ dessa Resolução, entendendo que os Objetos Intermediários de Concepção de um Programa de Pós-Graduação podem ser instrumentos associativos para a criação de outros Programas (Monteiro, in GRIMALDI, 2020).

Os objetivos propostos pelas formas associativas são: 1) a consolidação e expansão das áreas do conhecimento; 2) a redução das assimetrias territoriais; 3 ) viabilização de programas em formação por meio da parceria com programas consolidados (Art. $2^{\circ}$ da Portaria no 214 da CAPES).

Compreende-se que, para que os Programas de PósGraduação consolidados possam atuar nas formas associativas, de acordo com o proposto pela Portaria da CAPES, estes devem desenvolver ferramentas, identificar estruturas e atuar em condições de atribuir para as suas ações as bases necessárias para o alcance desses objetivos, e isso de acordo com realidades organizacionais e territoriais diversas.
O recorte apresentado nesse artigo considerou a existência de uma reciprocidade entre as formas associativas e os grupos de características intrínsecas e extrínsecas dos objetos intermediários de concepção compartilhados, e a partir dessa reciprocidade procurou-se discutir os impactos dessas interações sobre o alcance dos objetivos propostos pelas formas associativas, e por conseguinte sobre a viabilização de novos Programas (Figura 1).

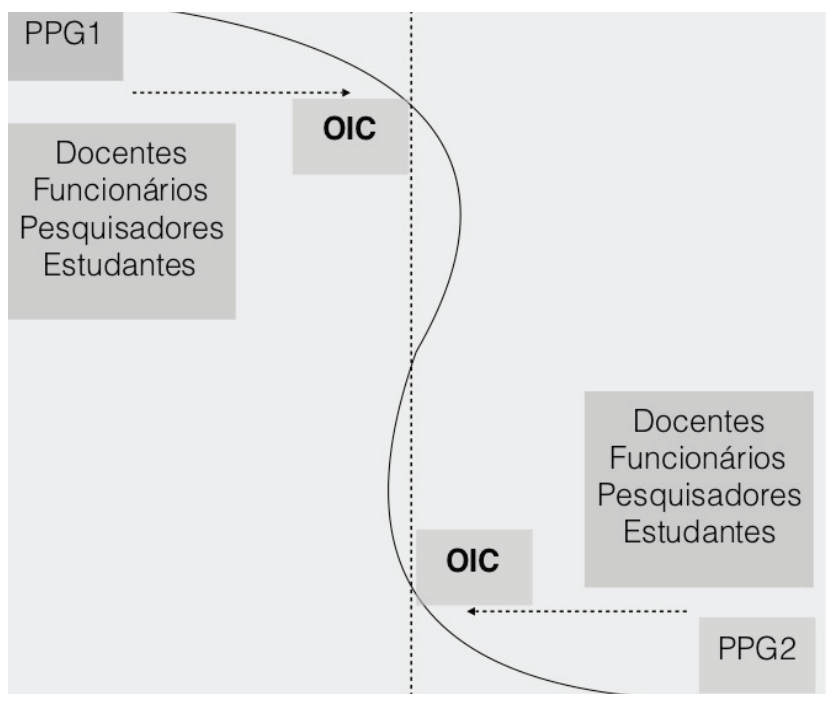

Figura 1 - Reciprocidade entre as formas associativas e os objetos intermediários de concepção Fonte: Autores

Reflete-se sobre a ampliação da abordagem dos Objetos Intermediários de Concepção, conforme tratada na disciplina Design Ecossocial, de acordo com o princípio de individuação apresentado por Simondon (2013), entendendo que o princípio de individuação dos objetos segundo suas caraterísticas intrínsecas e extrínsecas permite preservar a autonomia dos conhecimentos gerados em contextos diversos, reduzindo as desigualdades territoriais pelo fortalecimento de suas associações, adquirindo um papel de coordenador das formas associativas.

O meio associativo a ser considerado na Disciplina Design Ecossocial será definido como aquele no qual poderão interagir os pesquisadores, docentes, funcionários e estudantes de dois Programas de Pós-Graduação que estejam atuando em formas associativas. Na Figura 2 pode-se visualizar essa interação a partir de um dos Programas, no caso o Programa de Pós-Graduação em Design da EBA/UFRJ (Figura 2). 


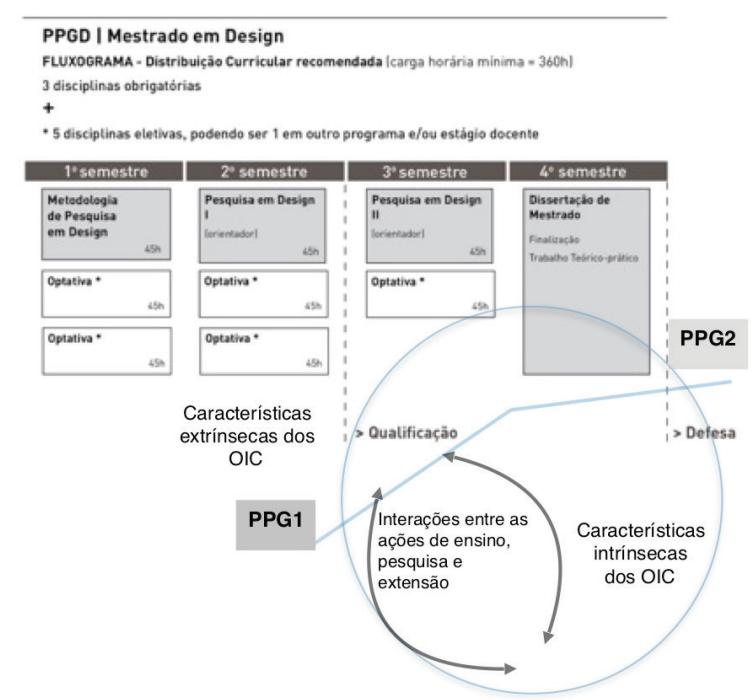

Figura 2: Levantamento das ações de ensino, pesquisa e extensão realizadas pelos Programas de Pós-Graduação associados

Fonte: Fluxograma do PPGD disponível em https://ppgd.eba.ufrj.br/estrutura-curricular)

O papel de cada um dos participantes na forma associativa será identificado com base na natureza dos Objetos Intermediários de Concepção produzidos por cada um desses participantes. Assim, mediação e coordenação são atuações a serem observadas no meio associativo e relacionadas aos objetos intermediários utilizados e gerados nas interações entre esses participantes (MER, JEANTET, TICHKIEWITCH, 1995).

\section{OBJETIVOS E METODOLOGIA}

O objetivo geral dessa pesquisa é desenvolver instrumentos didáticos e pedagógicos que permitam integrar os conhecimentos autônomos constituídos nas ações extensionistas ao ensino, à pesquisa e à extensão universitária no campo do Design. Entende-se como um desses instrumentos peda-gógicos as redes de compartilhamento, como os aplicativos educacionais (MONTEIRO, 2011).

Esse objetivo geral desdobra-se nos seguintes objetivos específicos:

1) consolidar e expandir a área de conhecimento do Design para a Inovação Social;

2) contribuir para reduzir as assimetrias territoriais tendo em vista o conhecimento da área de conhecimento específica;

3) viabilizar programas em formação por meio da experiência de criação e implementação do Programa coordenador da forma associativa.

A metodologia tem como referencia a Pesquisa-Ação, e mais especificamente os três movimentos identificados por ela: um que se realiza no sentido da pesquisa para a ação, que corresponde ao desenvolvimento do quadro teórico dos conhecimentos que amplia e consolida a prática profissional; outro que se realiza no sentido da ação para a pesquisa, que corresponde à interpretação dos resultados de uma intervenção prática no campo teórico do conhecimento; e um terceiro que se realiza num sentido convergente, com objetivos comuns, gerando conhecimentos com características autônomas em relação aos conhecimentos constituídos pelos dois movimentos anteriores (EL ANDALOUSSI, 2000; NICOLESCU, 1996; MORIN, 2010).

A metodologia da pesquisa, atualizada durante o pós-doutorado, está organizada em 4 etapas (Figura 3).

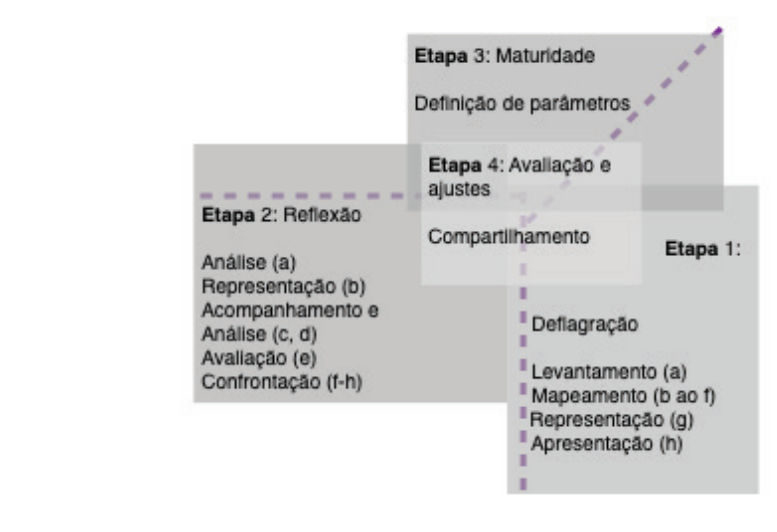

Figura 3 - Representação das etapas metodológias Fonte: Autores

O Essas etapas metodológicas têm como referência Melo (2020) e foram implementadas em dois projetos do Laboratório de Design e Inovação Social (LabDIS/EBA/UFRJ): o Pegada nas Escolas e o Design em Empreendimentos Populares (In: http://lidis.ufrj.br/\#projetos). São essas:

Etapa 1 - Deflagração

a) Levantamento das ações de ensino, pesquisa e extensão realizadas pelos Programas de Pós-Graduação associados.

b) Mapeamento dessas ações de acordo com as áreas temáticas de atuação para visualização preliminar de suas características intrínsecas. Idem para as características extrínsecas.

c) c) Idem para as características extrínsecas.

d) d) Mapeamento dos conhecimentos relativos ao projeto da ação e organização dos conjuntos de conhecimentos formados para a ação - características intrínsecas.

e) e) Idem para o conjunto de características extrínsecas.

f) f) Mapeamento dos conhecimentos autônomos, e 
organização do conjunto de conhecimentos formados na ação - - características intrínsecas e extrínsecas. g) Representação visual e gráfica dos mapeamentos e dos conjuntos organizados na forma de cenários característicos.

h) Apresentação e discussão com os interessados dos cenários característicos das ações de ensino, pesquisa e extensão e, no caso desta, com os interessados internos e externos à universidade.

i) Ajustes dos cenários característicos de acordo com os resultados das discussões.

Etapa 2 - Reflexão

a) Análise dos cenários caraterísticos com base nos conjuntos mapeados e de acordo com as áreas temáticas e contextos das ações, considerando possíveis desdobramentos.

b) Representação do cenário futuro possível das ações a partir dos cenários característicos.

c) Acompanhamento e observação das ações no campo.

d) Análise das observações.

e) Avaliação dos cenários futuros possíveis com os atores sociais (no campo).

f) Apresentação dos cenários característicos e dos cenários futuros possíveis destacando os conhecimentos formados pela ação, os conhecimentos para a ação e os conhecimentos autônomos.

g) Confrontação e debate com os participantes.

h) Análise dos resultados e representação dos requisitos e restrições para o desenvolvimento dos instrumentos didáticos e pedagógicos.

\section{Etapa 3 - Maturidade}

a) Definição e desenvolvimento dos objetos técnicos para integração à didática do ensino do design: associação de um conhecimento à uma operação, à um material e à um processo de fabricação (instrumentos didáticos)

b) Identificação de objetos intangíveis, relacionados ao processo de projeto: metodologias de projeto e parâmetros para inserção de um resultado num contexto (instrumentos pedagógicos).

Etapa 4 - Avaliação e ajustes

a) Desenvolvimento de instrumentos para acompanhamento e avaliação dos resultados: memória do projeto.

b) Compartilhamento dos resultados em rede virtual: App REDEsign UFRJ.

\section{REFERENCIAL TEÓRICO: A INFORMAÇÃO COMO OPERAÇÃO DE MEDIAÇÃO ENTRE A MATÉRIA E A FORMA}

Para alcançar o objetivo geral dessa pesquisa, de desenvolver instrumentos didáticos e pedagógicos que permitam integrar os conhecimentos autônomos constituídos nas ações extensionistas ao ensino, à pesquisa e à extensão universitária no campo do Design, tomou-se como base a Disciplina Design Ecossocial (BAP720), que é uma Disciplina eletiva do Programa de Pós- Graduação em Design (PPGD/EBA/UFRJ). O critério de seleção dessa disciplina foi a sua ementa:

A disciplina aborda o Design e a Inovação Social sob a ótica da Pesquisa-Ação e dos Objetos Intermediários de Concepção (OIC). Seu enfoque é a visualização como a operação de mediação, que dá forma ao Objeto a partir da convergência entre duas semi-cadeias técnicas: aquela que atualiza o potencial de plasticidade do material e aquela que define a estrutura da forma. A partir do estudo das formas, modos e graus de individuação dos objetos o Design é entendido como atividade relacional. O método consiste em considerar a relação como tendo um status de ser e a Inovação Social como transformação da realidade, na medida em que a essência do ser é conservada. A disciplina pertence à Linha de Pesquisa Imagem, Tecnologia e Projeto. (In: https://ppgd.eba.ufrj.br/horarios-e-ementas/)

Considera-se que o projeto de instrumentos produtores de objetos intermediários adequados à um ambiente de trabalho compartilhado precisa considerá-lo de forma indissociável como mode-lização da matéria e vetor de comunicação. Essa compreensão torna-se tão mais vital para o concepção compartilhada quanto mais ela estiver acompanhada de mudanças organizacionais, que se traduzem pela aproximação de diferentes conhecimentos profissionais. Nesse sentido o Design é entendido como uma atividade relacional, de acordo com a ementa da Disciplina Design Ecossocial, apresentada anteriormente.

De acordo com Mer, Jeantet e Tichkiewitch (1995), o conceito de Objeto Intermediário de Concepção vai além do encontro entre um material e uma forma. Ele é um elemento que permite coordenar o processo de concepção e portanto a própria atuação dos atores desse processo. Nesse ponto pode-se agrupar esses objetos de acordo com as suas características intrínsecas, que referem-se ao papel do ator como portador de conhecimentos 
específicos, e, em outro grupo, suas características extrínsecas, que remetem às condições de interação desse ator num processo de design.

Amplia-se a abordagem da natureza híbrida dos objetos intermediários de concepção de acordo com o princípio de individuação, tal como proposto por Simondon (2013): toda individuação supõe a existência de uma realidade anterior, chamada de pré individual, e que produz uma dupla que é composta pelo indivíduo e seu meio associativo. A realidade pré individual é o conjunto dos potenciais que a individuação vai atualizar na forma de uma estrutura mediada por uma singularidade. Uma vez ocorrida a individuação o indivíduo não está mais sozinho, ele é relativo à um meio associado, sendo esse meio uma reservatório de potenciais não atualizados.

A individuação opera-se como uma cristalização, como uma objetivação, que se dá a partir de uma tensão inicial entre duas ordens de grandeza incompatíveis. Para integrar-se numa determinada estrutura o ser pré individual vai se individualizar e essa individuação vai tornar-se potente a partir de uma mediação singular conferida por um objeto. Mas a realidade pré-individual não é um princípio nem um termo primário da individuação porque ela é um potencial, mas não atual (SIMONDON, 2013).

A realidade pré individual é uma relação de tensão entre duas ordens de grandeza sem comunicação antes da individuação e que a acompanha em todas as suas fases de atualização e se conserva sob forma de tensão na estrutura do indivíduo e sob a forma de potencial de ação no meio à ele associado (SIMONDON, 1958)

Igualmente, a singularidade não é um princípio nem é um indivíduo, ela existe a título de evento, quer dizer, em relação à uma situação e à um processo. Para Simondon (1958) todo indivíduo pode se individualizar novamente se as condições de meta-estabilidade estiverem reunidas.

Continuando com Simondon (2013) a experiência relativa à um mesmo objeto acrescenta e superpõe aspectos parcialmente contraditórios que produzem um estado meta-estável do saber relativo ao objeto. Nesse momento aparece um germe estrutural sob a forma de uma nova dimensão e observa-se uma estruturação que se prolonga pelo campo meta-estável, que é a experiência. Ocorre então a formalização. O estado de meta-estabilidade, que é entendido como aquele em que a experiência se formaliza, é caracterizado como sendo o estado em que a velocidade de transformação do objeto é tão fraca que parece que se aproxima de uma estabilidade.

O indivíduo representa à si próprio, se torna evento de sua própria historicidade, e se individualiza atualizando os potenciais do meio associado. Para pensar a realidade pela relação e pela informação o autor entende que a relação só é constitutiva para o ser e para o conhecimento se ela é ativa. A relação é centro ativo. Simondon (1958) dá o exemplo de um tijolo. Este não é o resultado da união de um material com uma forma, mas forma e matéria são resultados de uma operação técnica prévia na qual a forma é construída e a matéria é preparada.

L'homme prête alors sa propre individualité d'être vivant pour organiser cette opération; c'est en cela qu'il est porter d'outils. Par contre, lorsque l'objet technique est concrétisé, le mixte de nature et d'homme est constitué au niveau de cet objet; l'opération sur l'être technique n'est pas exactement un travail. En effet, dans le travail, I'homme coïncide avec une réalité qui n'est pas humaine, se plie à cette réalité, se glisse en quelque manière entre la réalité naturelle et l'intention humaine; I'homme, dans le travail, modèle la matière selon une forme; il arrive avec cette forme, qui est une intention de résultat, une prédétermination de ce qu'il faut obtenir au terme de l'ouvrage selon les besoins préexistants. Cette forme-intention ne fait pas partie de la matière sur laquelle le travail porte; elle exprime une utilité ou une nécessité pour l'homme, mais elle ne sort pas de la nature. L'activité de travail est ce qui fait le lien entre la matière naturelle et la forme, de provenance humaine; le travail est une activité qui arrive à faire coïncider, à rendre synergiques, deux réalités aussi hétérogènes que la matière et la forme. (SIMONDON, 1958, pp. 241-242)

A informação como encontro da matéria e da forma é, portanto, uma operação de mediação de duas semi-cadeias técnicas que fazem convergir a operação que atualiza o potencial de plasticidade do material e a operação que define a estrutura da forma (SIMONDON, 2013).

De acordo com Simondon (2013), o objeto é individualizado pela ação do homem e existe neste uma necessidade de individualizar os objetos, que se evidencia como um dos aspectos da necessidade de reconhecer-se e de encontrar-se num meio, e de se reencontrar como ser, tendo uma identidade definida, com um papel e uma atividade estáveis.

No entanto a individualização dos objetos não é absoluta mas relativa à uma expressão da existência psico-social do homem. Ela não pode, entretanto, ser arbitraria, mas precisa de um suporte que a justifique e que a receba.

Apesar da relatividade do princípio de individuação, em função de sua expressão como existência psico-social do homem, este princípio se sustenta em um dos aspectos 
dos objetos que ele considera como tendo uma única significação. Entretanto, esse aspecto assim reconhecido como tendo uma única significação, não estaria de acordo com a realidade que o contextualiza em função da exclusão dos outros pontos de vista, os quais poderiam se colocar para encontrar outros aspectos da individuação dos objetos na realidade. Para Simondon, é a atribuição única e exclusiva do princípio da individuação à tal ou tal tipo de realidade que é subjetiva, e essa condição não pode delimitar uma análise epistemológica e crítica desse princípio.

É preciso submetê-lo ainda ao estudo do conteúdo da noção de individuação para saber se ele exprime algo subjetivo, e se a dualidade entre as condições de atribuição desse princípio à forma ou à matéria se encontra no próprio conteúdo da noção de individuação.

Para entender o princípio da individuação é preciso colocar a questão sobre o que é a individuação. A partir dessa pergunta dois grupos são distinguidos por Simondon: o que é a individuação porque o indivíduo é o que ele é; e o que é a individuação porque o indivíduo é diferente de todos os outros e não pode ser confundido com eles.

No primeiro grupo a individuação é um conjunto de características intrínsecas, no segundo grupo um conjunto de características extrínsecas de relações. Mas como esses dois grupos podem ser relacionados um ao outro? Em qual ponto, em qual direção, o intrínseco e o extrínseco formam uma unidade?

Eles devem realmente ser separados ou devem indicar um modo de existência mais profundo, mais essencial, expresso nos dois aspectos da individuação? Mas, supondo que exista reciprocidade entre esses dois grupos, ou seja, entre o fato de um indivíduo ser o que é e o fato de que ser o que é o faz ser diferente dos outros, pode-se ainda dizer que o princípio de base é a individuação?

De acordo com Gilbert Simondon, o verdadeiro princípio deve ser descoberto no nível da compatibilidade entre $o$ aspecto positivo e o aspecto negativo da noção de individuação. Talvez então a representação do indivíduo deva ser modificada de acordo com o esquema da matéria que incorpora a informação.

A abordagem de Simondon apresenta uma espécie de ruptura com os modelos indutivos e dedutivos da ciência (DUHEM, 2015). A partir do estudo das formas, modos e graus da individuação ele recoloca o indivíduo no ser, segundo três níveis: físico, vital e psico-social. No lugar de supor substancias para entender a individuação ele considera os diferentes níveis de individuação como fundamentos dos domínios, tais como matéria, vida, espírito, sociedade. E ressalta que, para isso, é preciso um método e uma noção novos.
O método consiste em tentar não considerar a realidade por meio de uma relação conceitual entre dois termos extremos, e em considerar toda verdadeira relação como tendo um status de ser.

Assim, para Simondon (1958), num determinado sentido, o único princípio que pode nos direcionar é o princípio que conserva o ser no decorrer das suas transformações. Essa conservação existe através das trocas entre a estrutura e a operação, procedendo por saltos quânticos, através de equilíbrios sucessivos.

Para Simondon a forma só é forma se ela o é para o indivíduo. Ou seja, não existe, para o autor, forma do indivíduo que não seja, primeiramente, forma para o indivíduo. E é essa compreensão que vai permitir entender que, para o indivíduo, a relação tem valor de ser e que portanto o indivíduo não está em relação, e sim, ele é em relação. O princípio do indivíduo é o próprio indivíduo na sua atividade, que é relacional em si-mesma, como centro e mediação singular (SIMONDON, 2013).

O objeto-imagem é um intermediário entre o concreto e o abstrato quando ele condensa várias funções em unidade e emprega as solu-ções que estão associadas à essas funções na rede de realidades contemporâneas. Sua realidade de imagem é então paradigmática: ela permite compreender outras realidades conexas com as quais ela se articula e com as quais ela é solidária (SIMONDON, 2014).

\section{CONCLUSÃO}

Para aplicação desses princípios no contexto pesquisado, considera-se, como apresentado anteriormente, e de acordo com Monteiro (In GRIMALDI, 2020, p. 138) que:

Utilizando como base os conceitos de individuação, objetos intermediários de concepção e conhecimentos autônomos em Design entende-se que a conversação, o confronto e a corroboração de eventos que embasaram a criação do PPGD/ EBA/UFRJ compõe um todo coerente que integra diferentes narrativas e pontos de vista, que foram individualizados nos objetos intermediários de concepção compartilhados pelos participantes durante a criação desse Programa. Existe, portanto, um potencial que pode ser compartilhado numa possível atuação do Programa de acordo com as formas associativas. (...) A atuação nessas formas deverá implicar ainda, e de forma muito específica, na adaptação e transformação dessas ferramentas, estruturas e condições, de acordo com realidades organizacionais e territoriais diversas, e na preservação e fortalecimento da 
autonomia dos Programas envolvidos, independentemente de seu papel nas formas associativas. (MONTEIRO, in GRIMALDI, 2020, p. 138.

Ao relacionar as características intrínsecas, e as características extrínsecas relativas aos conhecimentos identificados no contexto de ensino, pesquisa e extensão dos Programas Associados, e estas características aos conhecimentos autônomos formados pelo movimento com sentido convergente dessas ações, entende-se que os conhecimentos autônomos parecem indicar para uma expansão do conjunto de conhecimentos relativos ao ensino, à pesquisa e à extensão universitária no campo do Design.

Essa expansão indica, a partir do referencial teórico-metodológico apresentado, para a demanda de desenvolvimento de instrumentos pedagógicos e de sua aplicação na didática do ensino, da pesquisa e da extensão no campo do Design. Olhar o Design e a Inovação Social na perspectiva das interações entre os atores sociais e os objetos projetados, nos seus contextos de interação, para entender como a ação é representada pelo pesquisador e como a abordagem prática refaz essa representação em sinergia com os atores, e no contexto da ação, é uma abordagem inovadora em relação ao ensino, à pesquisa e à extensão em Design, com a qual esse pesquisa está alinhada.

Destaca-se como relevante a inserção da extensão universitária no domínio do Design para a Inovação Social e a reflexão sobre o processo de emancipação e autonomia que o reconhecimento das próprias habilidades, sua formalização e disseminação têm sobre o ator do projeto e sobre os atores sociais que desempenham papeis convergentes com o papel do pesquisador na ação. A disciplina Design Ecossocial aborda essas questões nos níveis da Graduação e da Pós Graduação.

Com base no aprofundamento da pesquisa durante o período de pós-doutorado pode-se pensar na ampliação das fronteiras do ensino, da pesquisa e da extensão em Design de forma convergente com a atuação nas formas associativas, de acordo com a Portaria no 214, de 27 de outubro de 2017, da CAPES; reflete-se ainda sobre o desenvolvimento de estratégias que possam reverter a mercantilização das atividades universitárias e a alienação cultural, consequentes ao neoliberalismo; e sobre o estabelecimento e a consolidação das bases de cooperação no campo da extensão universitária visando sua internacionalização (THIOLLENT, 2016). As principais perspectivas de cooperação futura com o ÉCOLAB/ESAD/Orléans são:

- realização de cursos de pequena duração nos meses de janeiro e fevereiro de 2019 e 2020 na ÉSAD/Orléans, em colaboração com o Le Studium Loire Valley (em fase de discussão);
- convite para a participação do Dr. Ludovic Duhem como palestrante convidado 17th ERGODESIGN and 17th. USIHC - International Congress of Ergonomics, Usability and Human- Computer Interaction will be held from May 22 to May 24, 2019 in Rio de Janeiro, Brazil. Premilinary titles das apresentações de Dr DUHEM are : Lec-ture 1 for Ergodesign : "Theory of use in design. Some propositions about practical relations into a "milieu" analysis approach". And, Lecture 2 for USHIC : "Transduction as model for human- com-puter relation. The Simondon's thought in digital era."

- Acordo de cooperação internacional entre a Universidade Federal do Rio de Janeiro, através da Escola de Belas Artes, e The School of Higher Education in Art and Design de Orléans (ESAD ORLEANS);

- Desenvolvimento das bases de implantação do Laboratório Internacional de Extensão Universitária (LIEU).

\section{AGRADECIMENTOS}

- Ao Le Studium Loire Valley, Institute for Advanced Studies, um tesouro no coração do Val de la Loire.

- ÉCOLAB/École Supérieure d'Art et de Design d'Orléans, pela convivência enriquecedora.

- Escola de Belas Artes, Universidade Federal do Rio de Janeiro, pela oportunidade do exercício da Direção Adjunta de Extensão da EA/UFRJ.

\section{REFERÊNCIAS}

DUHEM, L. (2015) "Penser le numérique avec Simondon". In: https:/www.academia.edu/ 9024613/ Penser_le_numérique_avec_Simondon_Thinki ng_ the_digital_with_Simondon_Acesso em 18 de junho de 2018.

EL ANDALOUSSI, K. (2000) Recherches- actions. Sciences, Développement, Démocratie. Éditions Publisud, França.

FUNDAÇÃO COORDENAÇÃO DE APERFEIÇOAMENTO DE PESSOAL DE NÍVEL SUPERIOR, PORTARIA No 214, DE 27 DE OUTUBRO DE 2017 . DOU No 209, terça-feira, 31 de outubro de 2017. Seção 1 p. 35.

MONTEIRO, B., (2020) “Objetos Intermediários de Concepção: modelização, coordenação e comunicação em meios associativos". In GRIMALDI, M.(Org) Arquivos da Escola de Belas Artes. Rio de Janeiro, RioBooks, julho de 2020, pp. 137-151. Disponível em: https://bit.ly/2FQvR4L

MELO, V. A. de. (2000). Manual para Otimização da Utilização de Equipamentos de Lazer. SESC. MER, S. , JEANTET, A., TICHKIEWITCH, S., (1995) “Les 
Objets Intermédiaires de la conception : Modélisation et Communication". In: KHALDOUN, Z., CAELEN, J., Le communicationnel pour concevoir. Paris, Europia Productions.

MONTEIRO, B. G., (2011) "Conhecimentos Autônomos em Design: assimetrias de um campo de ação." Revista Interfaces, número 14, vol. I/ janeiro-julho de 2011.

MORIN, A. (2010) Cheminer ensemble dans la réalité complexe. La recherche-action intégrale et systémique (RAIS). Paris: L'Harmattan.

NICOLESCU, B. (1996) La transdisciplinarité. Manifeste. Monaco: Éditions du Rocher.

SILVA, A.L., MONTEIRO, B.G., (2009) "O design como ferramenta na área da educação: uma estratégia de inovação para sustentabilidade através da integração entre pesquisa, extensão e ensino num curso de graduação em Projeto de Produto." Anais do $2^{\circ}$ Simpósio Brasileiro de Design Sustentável (II SBDS). Rede Brasil de Design Sustentável - RBDS. São Paulo | Brasil | 2009. ISSN 21762384.

SIMONDON, G., (2013) L'individuation à la lumière des notions de forme et d'information. Grenoble, Éditions Jérôme Millon, 2013 (Primeira edição 2005). SIMONDON, G. (2014) Imagination et Invention. 1965-1966. Paris:PUF.

SIMONDON, G., (1958) Du mode d'existence des objets techniques. Paris, Aubier, Éditions Montaigne,1958.

THIOLLENT, M. (2016) "Por uma melhoria na extensão universitária". CCNEXT, Santa Maria.

\section{AUTORES}

ORCID: https://orcid.org/0000-0003-1596-5748

BEANY GUIMARÃES MONTEIRO, PROFESSORA D.SC. | Universidade Federal do Rio de Janeiro| Design | Rio de Janeiro, RJ Brasil | Correspondência para: R. Roberto Dias Lopes, 100/1204 - Leme, Rio de Janeiro - RJ, 22010110 | E-maill: beanymonteiro@eba.ufrj.br

\section{COMO CITAR ESTE ARTIGO}

MONTEIRO, Beany. Ensino, Pesquisa E Extensão Universitária Sob A Ótica Do Design Para Inovação Social. MIX Sustentável, [S.I.], v. 7, n. 1, p. 151-158, dez.2020.ISSN24473073. Disponível em:<http://www. nexos.ufsc.br/index.php/mixsustentavel>. Acesso em: dia mês. ano. doi:https://doi.org/10.29183/2447-3073. MIX2020.v7.n1.151-158.

DATA DE ENVIO: 20/09/2020

DATA DE ACEITE: 01/12/2020 